\title{
GROUND-BASED ASTROMETRY WITH SHORT-FOCUS CCD-ASTROGRAPHS
}

\author{
I.S. GUSEVA \\ Pulkovo Observatory \\ 196140 St Petersburg \\ Russia
}

\begin{abstract}
The main complication to use CCDs for astrometry is their small linear size. Nevertheless, a wide range of traditional astrometric problems can be solved by use of short-focus astrographs equipped with available CCDs. They are: creation of large astrometric and photometric catalogues, link of optical catalogues to an extragalactic reference frame, observations of the solar system bodies, etc. It can be shown that a telescope with focal length of 1-2 meters, equipped with a single CCD can produce a huge amount of simultaneous positional and photometric observations of high precision. It will be especially effective when the Tycho catalogue will be completed, because the problem of precise reference system in a small field of view (about 1 square degree) will be solved with extremely high accuracy. The majority of stars up to 18-20 magnitude can be observed with such an instrument. The problem of resolving "close" stars arises only for some very dense areas on the sky, such as globular clusters etc. - The results of CCD-observations made at Pulkovo with a small short-focus astrograph $(\mathrm{D}=100 \mathrm{~mm}, \mathrm{~F}=712 \mathrm{~mm}, \mathrm{FOV}=45 \times 67 \mathrm{arcmin})$ in 1993-1994 show that the precision of single observation is not worse than: in right ascension 0.15 to 0.35 arcsec, in declination -0.10 to 0.25 arcsec (the size of pixel was $18 \times 24 \mu \mathrm{m}^{2}$ ), and in magnitude -0.05 to $0.15 \mathrm{mag}$ in a wide range of magnitudes from the brightest objects to $\mathrm{V}=16$. From a series of $5-10$ frames it is possible to obtain positions and magnitudes of a large number (up to 500-600 in one frame) of stars with a precision quite comparable with that of normal astrographs and other "large" telescopes.
\end{abstract}

